\title{
Functional classification of esterases from leaves of Aspidosperma polyneuron M. Arg. (Apocynaceae)
}

\author{
Vanda Marilza de Carvalho, Rosimar Maria Marques, Ana Sílvia Lapenta \\ and Maria de Fátima P.S. Machado \\ Departamento de Genética e Biologia Celular, Universidade Estadual de Maringá, Maringa, \\ Paraná, Brazil.
}

\begin{abstract}
Polyacrylamide gel electrophoresis system (PAGE) and inhibition tests for biochemical characterization of $\alpha$ - and $\beta$-esterases were used to obtain a functional classification of esterases from Aspidosperma polyneuron. The characterization of $\alpha$ - and $\beta$-esterases from young leaves of $A$. polyneuron by the PAGE system showed fourteen esterase isozymes. The differential staining pattern showed that Est-2 isozyme hydrolyzes $\beta$-naphthyl acetate; Est-6, Est-7 and Est-8 isozymes hydrolyze $\alpha$-naphthyl acetate, and Est-1, Est-3, Est-4, Est-5, Est-9, Est-10, Est-11, Est-12, Est-13, and Est-14 isozymes hydrolyze both $\alpha$ - and $\beta$-naphthyl acetate. Inhibition pattern of $\alpha$ - and $\beta$-esterases showed that Folidol is a more potent inhibitor that Malathion, while Thiamethoxan (an insecticide with organophosphorus-like action) acts as an Est-4 and Est-6 inhibitor and induces the appearance of Est-5 and Est-7 isozymes as more intensely stained bands. Inhibition tests showed that OPC insecticides inhibit or activate plant esterases. Thus, plant esterases may be used as bioindicators to detect the presence and toxicity of residues of topically applied insecticides in agriculture and may be valuable for monitoring pollutants in the environment.
\end{abstract}

Key words: esterase classification, peroba-rosa, Ops, inhibition test.

Received: June 26, 2002; accepted: March 26, 2003.

\section{Introduction}

The isozymes $\alpha$ - and $\beta$-esterases can be identified by electrophoresis due to their in vitro preferences for $\alpha$ - and $\beta$-naphthyl ester substrates (Holmes and Masters, 1967; Coates et al., 1975). Furthermore, the most widely used functional classification of esterase activities relies primarily on their sensitivity to diagnostic concentrations of three groups of inhibitors, namely sulphydryl reagents (p-chloromercuribenzoate, $\mathrm{pCMB}$ ), organophosphates (OPs), and eserine sulfate (Holmes and Masters, 1967). The use of these inhibitors distinguishes four classes of esterases: acetylesterases, which are not affected by any of the inhibitors and generally prefer aliphatic substrates involving acetic acid; arylesterases, which are only inhibited by the sulphydryl reagents and generally prefer aromatic substrates; carboxylesterases, which are only inhibited by the OPs and prefer aliphatic esters, generally of longer acids than acetic acid; cholinesterases, which are inhibited by both OPs and eserine sulfate and prefer charged substrates, such as cholinesters, to other aromatic or aliphatic esters.

Send correspondence to Fátima P.S. Machado. E-mail: mfpsmachado@uem.br.
The inhibition tests for biochemical characterization of esterases from microbes (Lee et al., 1987; Oakeshott et al., 1993), vertebrates (Leibel, 1988; Higa et al., 1989; Lith et al., 1989; Deimiling and Wassmer, 1991) and insects, particularly species of Drosophila groups (Healy et al., 1991; Lapenta et al., 1995; 1998), have been important to decipher the different physiological processes in which esterases act. $\alpha$ - and $\beta$-esterases have been extensively studied in insects and are involved in different physiological processes, such as regulation of juvenile hormone levels (Kort and Granger, 1981), reproduction (Richmond et al., 1980; Mane et al., 1983), functioning of nervous system, and development of resistance to insecticides (Fournier et al., 1993; Guillemaud et al., 1997; Hemingway and Karunaratne, 1998). However, the functional characterization of plant esterases have been as yet scantly explored. The inhibition pattern of $\alpha$ - and $\beta$ - esterases in plants showed arylesterase and a differential expression of an esterase isozyme in young unexpanded leaves of cassava plants as a marker of pathogenesis after infection with bacteria (Pereira et al. 2001).

In the present study, inhibition tests for the biochemical characterization of esterases by native polyacrylamide 
gel electrophoresis were used to show its differential expression in leaves of Aspidosperma polyneuron after in vitro exposure to insecticides, and to obtain a functional classification of these esterases. The differential expression of esterases may be employed as a biosensor to detect the environmental pollution caused by insecticide compounds in plants.

\section{Materials and Methods}

In the state of Paraná, southern Brazil, the species Aspidosperma polyneuron M. Arg. (Apocynaceae) is known as "peroba-rosa". Samples of young leaves (8-10 mm long) of A. polyneuron were collected from native plants naturally grown for 54 years in the "Horto Florestal Dr. Luiz Teixeira Mendes", a 37-hectare Botanical Garden localized in the urban area of Maringá, northwestern region of Paraná (PR), Brazil. The "Horto Florestal" is a protected area for preservation of native and rare species which normally occur in small isolated or fragmented populations that have been reduced in size.

Electrophoretic analyses were carried out on samples consisting of individual young leaves collected from five 'peroba-rosa' tree. Four leaves of each tree were individually homogenized with a glass rod in an Eppendorf microcentrifuge tube using $100 \mu \mathrm{l}$ of $1.0 \mathrm{M}$ phosphate buffer, $\mathrm{pH}$ 7.0, containing 5\% PVP-40, $0.01 \mathrm{M}$ dithiothreitol (DTT), $10 \mathrm{mM}$ sodium metabisulfite, $50 \mathrm{mM}$ ascorbic acid, $1.0 \mathrm{mM}$ EDTA, and $0.5 \% \beta$-mercaptoethanol solution (Resende et al., 2000). After homogenization, the samples were centrifuged at $25,000 \mathrm{rpm}$, for $30 \mathrm{~min}$, at $4{ }^{\circ} \mathrm{C}$ in a Sorval $3 \mathrm{~K}-30$ centrifuge; supernatant $(20 \mu \mathrm{L})$ was used for each sample. Each leaf was replicated 4 times in the same gel which, after electrophoresis, was vertically divided into 5 parts for the control and the four inhibition tests.

The polyacrylamide gels $(12 \%)$ for vertical electrophoresis were prepared with $0.37 \mathrm{M}$ Tris- $\mathrm{HCl}$, and $\mathrm{pH} 8.8$ as buffer (Ceron, 1988). The stack gel was prepared with $3.0 \mathrm{~mL}$ of acrylamide $10 \%$ and bis-acrylamide $0.5 \%$ dissolved in $3.0 \mathrm{~mL}$ of $0.24 \mathrm{M}$ Tris- $\mathrm{HCl}, \mathrm{pH} 6.8,30 \mu \mathrm{L}$ twice-distilled water, $250 \mu \mathrm{L}$ ammonium persufate $2 \%$ and $30 \mu \mathrm{L}$ TEMED. Gels were run during $3 \mathrm{~h} 30 \mathrm{~min}$, at $25^{\circ} \mathrm{C}$, and constant $200 \mathrm{~V}$. The running buffer was $0.1 \mathrm{M}$ Tris-glycine, $\mathrm{pH}$ 8.3. Staining techniques by Johnson et al. (1966) and Steiner and Johnson (1973), modified by Ceron (1988), were used for esterase identification. The gels were soaked for $30 \mathrm{~min}$ in $50 \mathrm{~mL} 0.1 \mathrm{M}$ sodium phosphate, $\mathrm{pH}$ 6.2 , at room temperature. Esterase activity was visualized by placing the gels for $1 \mathrm{~h}$ in a staining solution prepared with $50 \mathrm{~mL}$ of sodium phosphate solution, $15 \mathrm{mg}$ of $\beta$-naphthyl acetate, $20 \mathrm{mg}$ of $\alpha$-naphthyl acetate, $60 \mathrm{mg}$ of Fast Blue RR salt, and $5 \mathrm{~mL}$ of N-propanol.

Inhibition tests for the biochemical characterization of esterases involved respectively $0.01 \mathrm{~g}(0.02 \mathrm{mM})$ p-chloromercuribenzoate (pCMB), $30 \mu \mathrm{L}(1.6 \mathrm{mM})$ Mala- thion, $30 \mu \mathrm{L}(51 \mathrm{mM})$ Folidol 500, or $0.5 \mathrm{mg}(0.01 \mathrm{mM})$ Thiamethoxam $250 \mathrm{WG}$, each prepared with $100 \mathrm{~mL}$ twice-distilled water. These compounds were used separately in the pre-incubation and staining solutions of the different sections of each gel. Malathion and methylParathion (the active substance of Folidol 500) are organophosphate pesticides extensively used to control a wide range of sucking and chewing pests in crops, fruits, and vegetables. Thiamethoxan insecticide is a new, secondgeneration product of a neonicotinoid compound of low toxicity used in crops to control sucking insects (Novartis Biociências S.A.); the neonicotinoid compound acts in a similar way as organophosphates. pCMB being an arylesterase inhibitor, while Malathion and MethylParathion (OP compounds or OPCs) are carboxylesterase and cholinesterase inhibitors (Healy et al., 1991).

Polyacrylamide gels were dried, as described by Ceron et al. (1992) and Lapenta et al. (1995). Gels were kept at room temperature and fixed for $1 \mathrm{~h}$ in a mixture of $7.5 \%$ acetic acid and $10 \%$ glycerol embedded in 5\% gelatin, placed between two sheets of wet cellophane paper stretched on an embroidering hoop and left to dry for $24-48 \mathrm{~h}$.

\section{Results}

Fourteen esterase isozymes were detected in leaves of the five analyzed plants of $A$. polyneuron when vertical polyacrylamide gel was used. The esterases were numbered in sequence starting from the anode, according to their decrease in negative charge (Figure 1A). Different esterase bands present characteristic colors, that is: $\alpha$ (black), $\beta$
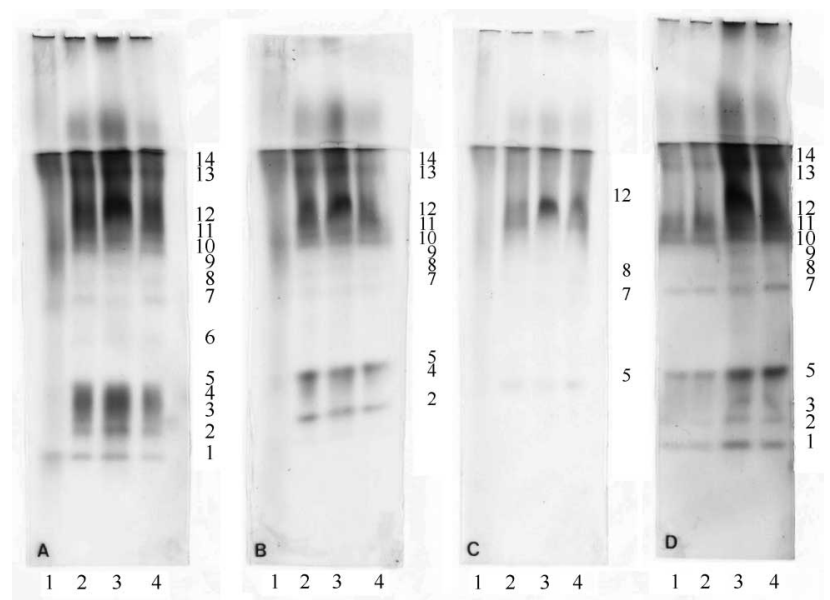

Figure 1 - Inhibition pattern for characterization of esterases from leaves of Aspidosperma polyneuron showing that Est-1, Est-3, Est-4, and Est-6 isozymes were inhibited in the presence of Malathion (B); Est-5, Est-7, Est-8, and Est-12 isozymes were detected as weakly stained bands by Folidol (C); Est-3, Est-4, and Est-6 isozymes were inhibited by Thiamethoxan (D). The gel in A shows $\alpha$ and $\beta$ esterases in the absence of inhibitors. Lanes 1-4 correspond to leaf samples of different plants of $A$. polyneuron. 
(red), and $\alpha \beta$ (deep purple), since they hydrolyze different substrates, respectively: $\alpha$-naphthyl acetate, $\beta$-naphthyl acetate, and both $\alpha$ - and $\beta$-naphthyl acetate. The staining specificity of each band was clearly determined by exposing gels to staining solutions containing only $\alpha$-naphthyl acetate or/and $\beta$-naphthyl acetate; they were: Est-2 (red), Est-6, Est-7 and Est-8 (black), and Est-1, Est-3, Est-4, Est-5, Est-9, Est-10, Est-11, Est-12, Est-13, and Est-14 (deep purple), (gels not shown).

The inhibition pattern of $\alpha-, \beta$ - and $\alpha \beta$-esterases from A. polyneuron showed that no esterase isozyme has been inhibited by pCMB (gels not shown). Est-1, Est-3 and Est-6 isozymes were inhibited by Malathion (Figure 1B). The bands of Est-5, Est-7, Est-8 and Est-12 isozymes presented weak staining (partial inhibition), while all other Est isozymes were inhibited by Folidol (Figure 1C). Est-4, and Est-6 isozymes were inhibited by insecticide Thiametoxan, whereas Est-5 and Est-7 isozymes presented the most intensely stained bands (Figure 1D).

\section{Discussion}

PAGE and inhibition tests for biochemical characterization of esterases from leaves of $A$. polyneuron showed one $\beta$-esterase (Est-2), three $\alpha$-esterases (Est-6, Est-7 and Est-8), ten $\alpha \beta$-esterases (Est-1, Est-3, Est-4, Est-5, Est-9, Est-10, Est-11, Est-12, Est-13 and Est-14), and a selective sensitivity to organophosphate compounds for different esterases in these plants. Fourteen esterase isozymes have been detected by PAGE in leaves of different cassava cultivars (Pereira et al., 2001). The number of esterases identified in the two plant species seems to be lower than the number of esterases detected in similar studies of mammalian and insect species (Oakeshott et al., 1993; Lapenta et al., 1998). Functional characterization of esterases from other species and from different tissues may be important to determine the actual number of different: $\alpha$-, $\beta$-, and $\alpha \beta$-esterases in plants. Analyses of different tissues may reveal a larger number of esterases in plants. Esterase sensitivity to OPCs has been described in animals (Kaliste-Korhonen et al., 1996; Cabello et al., 2001), albeit their effect has not been explored in plants. Our results showed that Malathion and Methyl Parathion, used to control insect pests, were also able to inhibit $\alpha$-, and $\alpha \beta$-esterases in leaves of $A$. polyneuron. These results are consistent with previously observations that significant similarities exist between most eukariotic carboxylesterases and all the eukaryotic cholinesterases sequenced to date (Oakeshott et al., 1993; Mikhailov and Torrado, 2000). These enzymes are consequently placed in a single carboxyl/cholinesterase multigene family (Oakeshott et al., 1999). It has been reported that the sequences required for the hydrolytic activity of carboxylesterases, acetylcholinesterases and cholesterol-esterases have been evolutionarily conserved to a high degree (Satoh and Hosokawa, 1998).
Cholinesterases or esterases with functional activity similar to cholinesterase have not been reported in plants. On the other hand, cholinesterases with non-cholinergic functions have been described in vertebrates. Such isozymes are termed nonspecific cholinesterases or pseudocholinesterases (Chatonnet and Lackridge, 1989). Thus, we propose the hypothesis that $A$. polyneuron esterases inhibited by Folidol represent examples of the plant's nonspecific cholinesterases that primarily have a noncholinergic function in leaf tissues. It has been suggested that cholinesterases may have a proteolytic activity in addition to its cholinergic activity, acting as proteases, to regulate cell growth and development (Small, 1990). Carboxylesterase activity in plants has been correlated with the differentiation processes (Gahan et al., 1983; Melati et al., 1996) and inhibition of carboxylesterases was employed as a biosensor for detecting selenium compounds in Thevetia peruviana seeds (Saritha and Nanda Kumor, 2001).

We detected in A. polyneuron leaves a differential inhibition pattern for esterases by OPCs. Folidol acted as a more potent inhibitor than Malathion, while Thiamethoxan (an insecticide with an organophosphate-like action) acted as an Est-4 and Est- 6 inhibitor and induced the enhancement of Est-5 and Est-7 bands staining intensities. This selective sensitivity of esterase to organophosphate compounds favors its binding to different OPCs. It has been proposed that, in the process of specific OPC detoxification, carboxylesterases participate by binding OPCs to their active center and sequestering them in the cell, or hydrolyzing the ester bonds of OPC molecules (Jakonovic et al., 1996; Tang and Chambers, 1999). The not yet explained action of Thiamethoxan inducing the more intensely stained bands has also been detected in inhibition tests for certain species of insects (Ruvollo-Takasusuki et al. and Lapenta et al., unpublished results).

The inhibition tests for biochemical and functional classification of esterases by PAGE in the current study were important since they differentiated the esterases of $A$. polyneuron leaves in $\alpha-, \beta$-, and $\alpha \beta$-esterase, according to their substrate preferences, and showed that OPC insecticides were able to act by inhibiting or activating plant esterases. Therefore, it is possible that plant esterases may be used as bio-indicators to detect the presence and toxicity of insecticide residues topically applied in agriculture and may be valuable for monitoring pollutants in the environment. Manwell and Baker (1968) suggested that pollutants may influence biochemical polymorphism, since some enzymes (e.g. esterases) interact directly with pesticides and other pollutants. Detected differential expression of esterase activity in plant leaves after incubation with insecticide compounds indicates the importance of further investigations to research the effect of different types and concentrations of these compounds during field application to different plant species. 


\section{References}

Cabello G, Valenzuela M, Vilaxa A, Duran V, Rudolph I, Hrepic $\mathrm{N}$ and Calaf G (2001) A rat mammary tumor model induced by the organophosphorous pesticides parathion and malathion, possibly through acetylcholinesterase inhibition. Environ Health Perpect 109:471-479.

Ceron CR (1988) Padrão de esterases no desenvolvimento de Drosophila mulleri, D. arizonensis e seus híbridos. Doctoral Thesis, Instituto de Biociências, Universidade de São Paulo, $142 \mathrm{p}$.

Ceron CR, Santos JR and Campos Bicudo HEM (1992) The use of gelatin to dry cellophane wound slab gels in an embroidering hoop. Brazil J Genet 15:201-203.

Coates PM, Mestriner MA., Hopkinson DA (1975) A preliminary genetic interpretation of the esterase isozymes of human tissues. Ann Hum Genet 39: 1-20.

Chatonnet A and Lockridge O (1989) Comparison of butyrylcholinesterase and acetylcholinesterase. Biochem J 260:625-629.

Deimilling O and Wassmer B (1991) Genetic characterization of esterase-28 (ES-28) of the house mouse. Biochem Genet 29:55-64.

Fournier D, Mutero A, Pralavorio M and Bride JM (1993) Drosophila acetylcholinesterase mechanism of resistance to organophosphates. Chem Biol Interations 87:233-238.

Gahan PB, Rana MA and Phillips R (1983) Activation of carboxylesterases in root cortical parenchymal cells of Pisum sativum during xylem induction in vitro. Cell Biochem Funct 1:109-111.

Guillemaud T, Makate N, Raymond M, Hirst B, and Callghan A (1997) Esterase gene amplification in Culex pipiensis. Insect Mol Biol 6:319-327.

Healy MJ, Dumancic MM and Oakeshott JG (1991) Biochemical and physisological studies of soluble esterases from Drosophila melanogaster. Biochem Genet 29:365-388.

Hemingway J and Karunaratne SHPP (1998) Mosquito carboxylesterase: a review of the molecular biology and biochemistry of a major insecticide resistance mechanism. Med Vet Entomol 12:1-12.

Higa HH, Manzi A and Varki A (1989) 0-Acetylation and de-0acetylation of sialic acids. Purification, characterization, and properties of a glycosylated rat liver esterase specific for 9-0-acetylated sialic acids. Biol Chem 264:435-442.

Holmes RS and Masters CJ (1967) The developmental multiplicity and isoenzyme status of cavian esterases. Biochim Biophys Acta 132:379-399.

Johnson FM, Kanapi CG, Richardson RH, Wheeler MR and Stone WS (1966) An operational classification of Drosophila esterases for species comparison. Univ Texas Publ 6615:517-532.

Jokanovic M, Kosanovic M and Maksimovic M (1996) Interaction of organophosphorus compounds with carboxylesterases in the rat. Arch Toxicol 13:261-268.

Kaliste-Korhonen E, Tuovinen K and Hanninen O (1996) Interspecies differences in enzymes reacting with organophosphates and their inhibition by paraoxon in vitro. Hum Exp Toxicol 15:972-978.

Kort CA and Granger NA (1981) Regulation of the juvenile hormone titer. Ann Rev Entomol 26:1-28.

Lapenta AS, Campos Bicudo HEM, Ceron CR and Cordeiro JA (1995) Esterase patterns of species in the Drosophila buzzatii cluster. Cytobios 84:13-29.
Lapenta AS, Campos Bicudo HEM, Ceron CR and Cordeiro JA (1998) Esterase patterns and phylogenetic relationships of species and strains included in the Drosophila buzzatii cluster. Cytobios 96:95-107.

Lee H, To RJB and Latta RK (1987) Some properties of extracellular acetylxylan esterase produced by the yeast Rhodotorula mucilaginosa. App Environ Microbiol 53:2831-2834.

Leibel WS (1988) An analysis of esterase activities from surgeonfish tissue yields evidence of atypical pseudocholinesterase. Comp Biochem Physiol 91B:437-447.

Lith HA, Bieman M nd Zutphen BFM (1989) Purification and molecular properties of rabbit liver esterase ES-1A. Eur J Biochem 184:545-551.

Mane SD, Tompkins L and Richmond RC (1983) Male esterase-6 catalyzes the synthesis of a sex pheromone in Drosophila melanogaster females. Science 222:419-421.

Manwell C and Baker CMA (1968) Genetic variation of isocitrate, malate and 6-phosphogluconate dehydrogenase in snails of the genus Cepaea - introgressive hybridization, polymorphism and pollution? Comp Biochem Physiol 26:195-209.

Melati MR, Scialabba A and Bellani ML (1996) Carboxylesterase activity in the carrot seed and seedling. Eur J Histochem 40:159-166.

Mikhailov AT and Torrado M (2000) Carboxylesterases moonlight in the male reproductive tract: a functional shift pivotal for male fertility. Front Bioscience 5:53-62.

Oakeshott JG, Claudianos C, Russel RJ and Robin GC (1999) Carboxyl/cholinesterases: a case study of the evolution of a successful multigene family. BioEssays 21:1031-1042.

Oakeshott JG, van Papenrecht EA, Boyce TM, Healy MJ and Russell RJ (1993) Evolutionary genetics of Drosophila esterases. Genetics 90:239-268.

Pereira AJ, Lapenta AS, Vidigal-Filho PS and Machado MFPS (2001) Differential esterase expression in leaves of Manihot esculenta Crantz infected by Xanthomonas axonopodis pv. manihotis. Biochem Genet 39:289-296.

Resende AG, Vidigal-Filho PS and Machado MFPS (2000) Isozyme diversity in cassava cultivars (Manihot esculenta Crantz). Biochem Genet 38:203-215.

Richmond RC, Gilbert DG, Sheehan KB, Gromko MH and Butterworth FM (1980) Esterase 6 and reproduction in Drosophila melanogaster. Science 207:1483-1485.

Saritha K and Nanda Kumar NV (2001) Qualitative detection of selenium in fortified soil and water samples by a paper chromatographic-carboxyl esterase enzyme inhibition technique. J Chromatogr A 919:223-228.

Satoh T and Hosokawa M (1998) The mammalian carboxylesterases: from molecules to functions. Ann Rev Pharmacol Toxicol 38:257-288.

Small DH (1990) Non-cholinergic actions of acetylcholinestases: proteases regulating cell growth and development? Trends Biochem Sci 15:213-216.

Steiner WWM and Johnson WE (1973) Techniques for electrophoresis of Hawaiian Drosophila US - IBP. Island Ecosyst. Tech Rep 30:1-21.

Tang J and Chambers JE (1999) Detoxification of paraoxon by rat liver homogenate and serum carboxylesterases and A-esterases. J Biochem Mol Toxicol 13:261-268. 